\title{
Evaluación del impacto de la reforma del sector de la salud en Costa Rica mediante un estudio cuasiexperimental
}

\author{
Luis Rosero Bixby ${ }^{1}$
}

Forma de citar

Rosero Bixby L. Evaluación del impacto de la reforma del sector de la salud en Costa Rica mediante un estudio cuasiexperimental. Rev Panam Salud Publica. 2004:15(2):94-103.

RESUMEN Objetivo. Valorar el impacto de la reforma del sector de la salud iniciada en Costa Rica en 1995 sobre la mortalidad de niños y adultos y la equidad en el acceso a los servicios de salud. Métodos. La reforma del sector de la salud se inició en Costa Rica en 1995 en algunos distritos, pero en otros su adopción fue posterior. Esto permitió efectuar un análisis de series temporales, usando un diseño cuasiexperimental con observaciones anuales de 1985 a 2001 en cada uno de los 420 distritos que tenía Costa Rica en 1984. Las series temporales se dividieron en tres períodos que permitieron agrupar a los distritos en tres categorías (grupo pionero, intermedio y tardío) según el momento en que iniciaron el proceso de reforma: 1995-1996; 1997-2000; 2001 o después, respectivamente. Las series incluyeron datos de defunción desagregadas por sexo, grupos de edad y tres grandes grupos de causas de muerte (enfermedades transmisibles, de origen social, o crónicas); estimaciones de población por edad y sexo; dos variables que miden la intervención (si el distrito ha adoptado o no la reforma $y$, en caso de que sí, el número de años desde que adoptó la reforma); y ocho variables para controlar efectos de confusión. Los datos se obtuvieron de los registros de defunciones y nacimientos, censos de población y estimaciones demográficas. Se usaron modelos de regresión múltiple de Poisson con efectos fijos para estimar el impacto de la reforma en la mortalidad. También se valoró el acceso de la población a los servicios de salud en el primer nivel de atención antes y después de la reforma. El indicador de acceso utilizado fue el porcentaje de personas que vivian a menos de 4 $\mathrm{km}$ de distancia de un servicio que ofreciera consultas médicas.

Resultados. Según lo estimado por los modelos de regresión, la adopción de la reforma redujo significativamente la mortalidad de los niños en $8 \%$ y la de los adultos en $2 \%$. El efecto fue de $14 \%$ en la mortalidad de adultos debida a enfermedades transmisibles o desencadenadas por procesos infecciosos, nulo en la debida a causas sociales y similar al total (2\%) en la mortalidad debida a enfermedades crónicas. Se estima que la reforma salvó aproximadamente 120 vidas de niños y 350 vidas de adultos en el año 2001 únicamente. La reforma se puso en marcha primero en las zonas menos densamente pobladas y de menor desarrollo socioeconómico. Como resultado, la brecha en la equidad del acceso a los servicios de salud en el primer nivel de atención se redujo significativamente. El porcentaje de personas sin acceso equitativo a los servicios de salud del primer nivel de atención se redujo en 15\% entre 1994 y 2000 en las áreas que adoptaron la reforma en 1995-1996, mientras que en las áreas que no habían adoptado la reforma en 2000, la reducción fue solo de $3 \%$.

1 Centro Centroamericano de Población, Universidad de Costa Rica, San José 2060, Costa Rica. Fax:
(506) 207-4809. Correo electrónico: Lrosero@ccp.

ucr.ac.cr 
Conclusión. La reforma redujo significativamente la mortalidad en Costa Rica y puso fin a una década de estancamiento en algunos indicadores, como la esperanza de vida. El efecto de la reforma se produjo probablemente gracias a su focalización en las regiones menos desarrolladas del país, lo cual dio lugar a un mejoramiento en la equidad de acceso al primer nivel de atención médica.

Palabras clave Reforma del sector de la salud, evaluación de impacto, mortalidad, equidad en el acceso, Costa Rica.

Costa Rica ha alcanzado un excelente nivel de salud y su esperanza de vida al nacer (77,7 años) es la más alta del continente americano después de Canadá (1) y supera en 0,8 años a la de los Estados Unidos de América (2) y en 2 años a la de Cuba, Puerto Rico y Chile. El sistema sanitario costarricense y sus logros han sido objeto de amplios análisis (3-4).

A pesar de estos logros, el excepcional avance a lo largo de varias décadas se estancó entre 1990 y 1995, lo que hizo pensar que el país había alcanzado sus máximas posibilidades (figura 1). Durante ese lustro, la esperanza de vida al nacer disminuyó de 76,9 a 76,2 años (5). Sin embargo, esa tendencia adversa pudo revertirse y en 2000 la esperanza de vida aumentó a 77,7 años. Si bien este aumento de 1,5 años alcanzado en un quinquenio no parece tan espectacular como el observado en las décadas de 1940 y 1970, constituye un hecho muy meritorio, dado lo difícil que resulta elevar este indicador más allá de los niveles que ya se habían alcanzado en Costa Rica. Este logro coincidió con la adopción del programa de reforma del sector de la salud en ese país. ¿Es esta coincidencia temporal una mera asociación fortuita? o, por el contrario, ¿puede establecerse un nexo causal entre la reforma y la reanudación del progreso?

La aplicación de la reforma del sector de la salud costarricense se inició en 1995 (aunque su planificación y los cambios administrativos se iniciaron varios años antes) y aún no había concluido a finales del año 2003. Los objetivos previstos por la reforma eran ambiciosos y diversos $(6,7)$. No obstante, en la práctica los esfuerzos se enfocaron hacia objetivos económicos, como mejorar la eficiencia y la racionalización en la asignación de recursos (8). Esto se debe a que este proceso de reforma se originó en gran medida en préstamos del Banco Mundial, lo que trajo consigo una participación muy activa de economistas. Entre las piezas clave de la reforma en la búsqueda de una mayor eficiencia se encuentran los llamados "compromisos de gestión", documentos que suscriben los gerentes locales de la gestión de salud (directores de áreas, de clínicas y hospitales) en el que se establecen metas e indicadores de progreso que sirven para la asignación de recursos y el posterior seguimiento de la gestión. Otra pieza clave de la reforma fue el establecimiento de los denominados equipos básicos de atención integral de la salud (EBAIS) para prestar servicios en el primer nivel de atención y lograr una mayor efectividad. Los EBAIS son equipos formados por uno o varios médicos y otros trabajadores de la salud. Este equipo es responsable de la salud de un sector bien delimitado que abarca a alrededor de 4000 personas. Originalmente, los EBAIS se implantaban en establecimientos de salud ya existentes. Sin embargo, este concepto evolucionó y los EBAIS se convirtieron en los últimos años en paquetes completos de recursos humanos e instalaciones, de modo que los EBAIS creados más recientemente se han establecido con su propia planta física $\mathrm{y}$, por lo tanto, constituyen un nuevo tipo de establecimiento de salud.

La mayor parte de los cambios introducidos por la reforma en la prestación de servicios tuvieron lugar en el

FIGURA 1. Tasa de mortalidad infantil (TMI) y esperanza de vida (EV). Costa Rica, 1970-2001

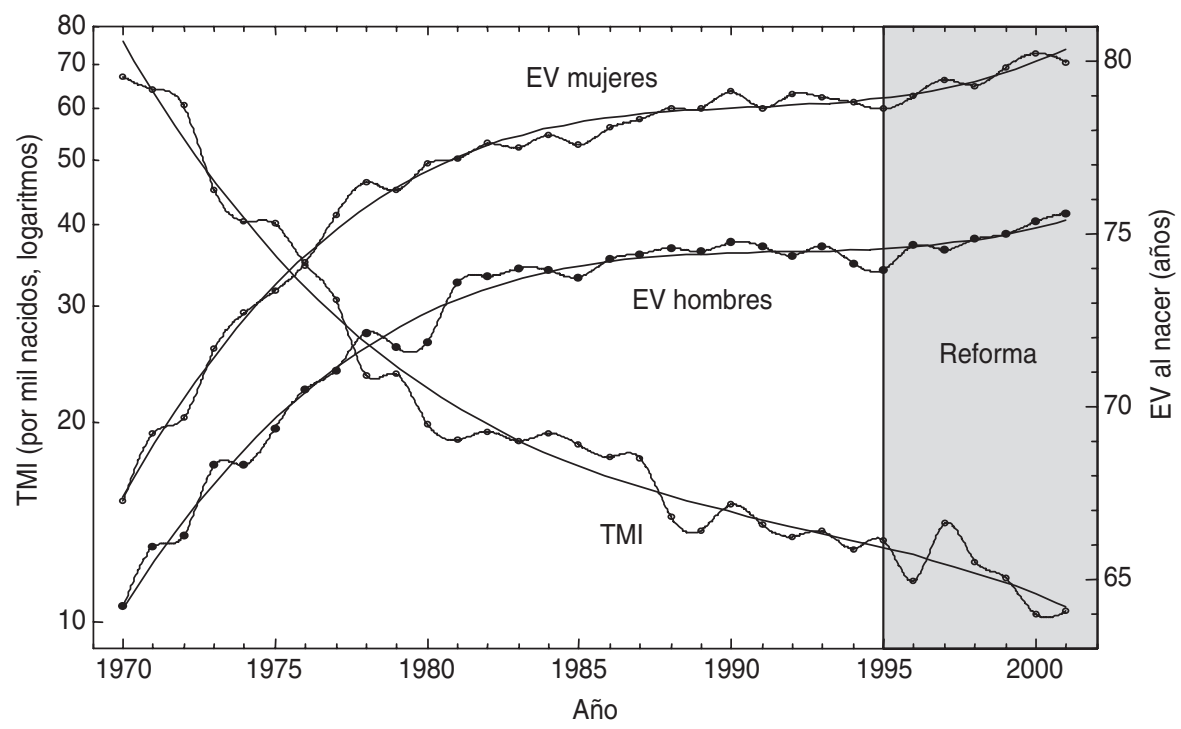


FIGURA 2. Mapa ilustrativo del proceso de adopción de la reforma de salud en Costa Rica, 1995-2001

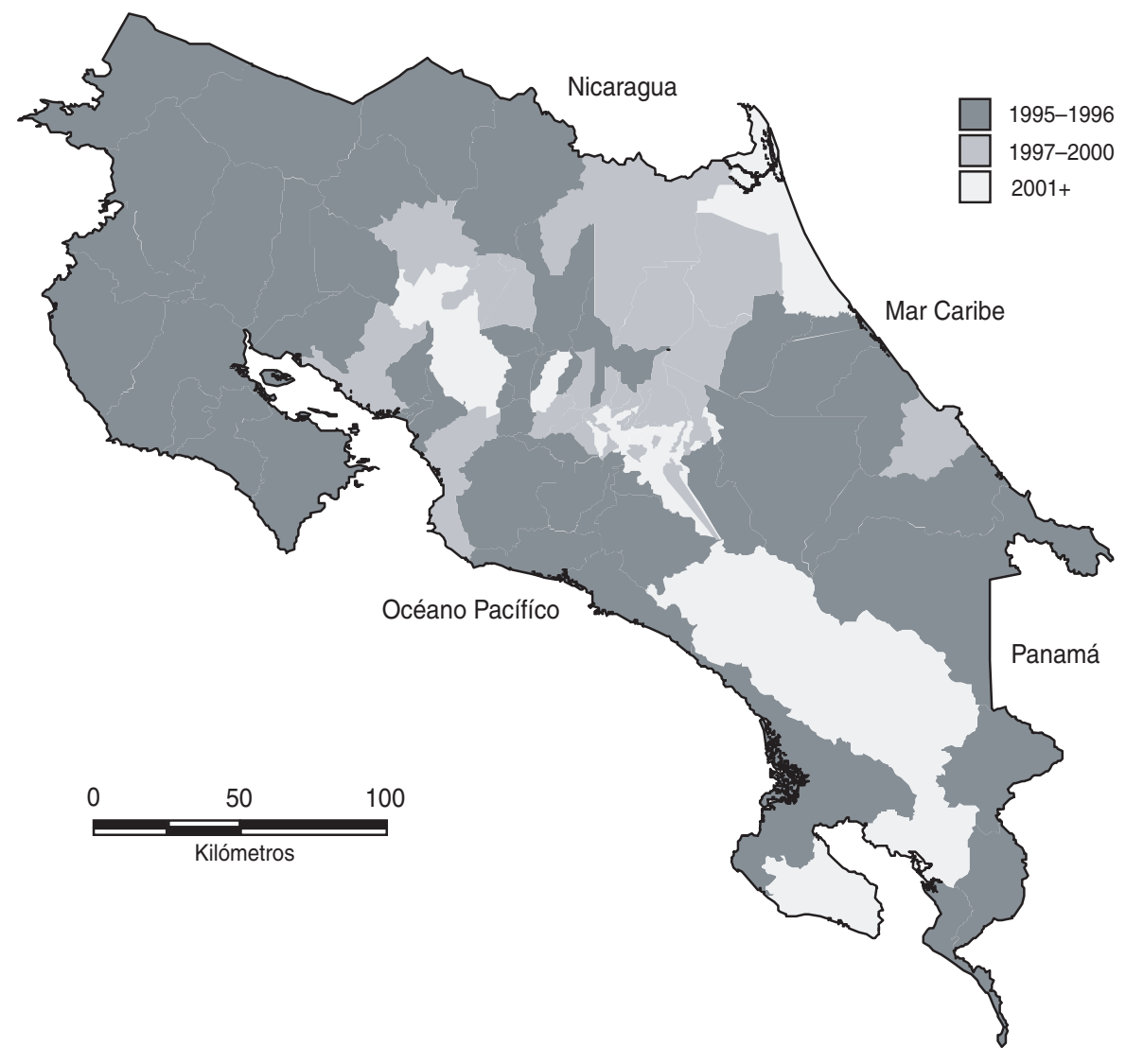

primer nivel de atención. Hasta el momento, las reformas en los hospitales han estado centradas principalmente en aspectos gerenciales, con la idea de lograr así una mejoría en la calidad de la atención sanitaria. La transferencia de establecimientos del Ministerio de Salud a la Caja Costarricense de Seguro Social (CCSS), efectuada como parte de la reforma, cambió sustancialmente la forma de brindar los servicios en el primer nivel de salud: numerosos centros y puestos de salud se cerraron por considerarse redundantes o se dedicaron a otras funciones, se abrieron establecimientos de atención primaria como consultorios comunales, se reorganizó la provisión de servicios en los establecimientos existentes y se crearon los EBAIS.

La reforma en los servicios de atención primaria de salud no ocurrió al unísono en todo el territorio nacional. personas y en la equidad de su acceso a los servicios de salud.

\section{MATERIALES Y MÉTODOS}

Se utilizó un diseño cuasiexperimental (11) con mediciones de resultados antes y después de la adopción de la reforma del sector de la salud (intervención) en diferentes áreas del país. En la evaluación se utilizaron dos procedimientos analíticos para neutralizar los posibles sesgos derivados de aplicar un diseño sin una aleatorización experimental completa: 1) la técnica de la regresión múltiple para el control estadístico de los efectos de variables de confusión (por ejemplo, la propensión de un área a innovar, o la propensión de otra a tener un deficiente desempeño en salud), que es equivalente a comparar solo comunidades con factores confusores similares; 2) la inclusión de mediciones antes y después de la intervención a fin de comparar tendencias o cambios (en vez de niveles), lo que permite depurar la comparación de peculiaridades de la comunidad presentes en todo el período en estudio (por ejemplo, un subregistro de defunciones o condiciones ambientales favorables para la salud); esto último se conoce en la jerga de la regresión para datos de tipo panel como modelos de "efectos fijos".

La base de datos para la evaluación consistió en un panel de series temporales con observaciones anuales entre 1985 y 2001 en los 420 distritos que existían en Costa Rica durante el censo de 1984. El distrito es el tercer nivel de la división políticoadministrativa del país, después de la provincia y el cantón, y por lo general es una unidad geográficamente homogénea de 5000 a 10000 habitantes.

Para construir las series temporales se tomó en cuenta que el número de distritos pasó de 420 a 459 durante el período estudiado. Sin embargo, se pudo reconstruir la división distrital de 1984 debido a que la gran mayoría de los nuevos distritos se originaron íntegramente a partir de un distrito único anterior. En los pocos casos en que un nuevo distrito se originó a par- 
tir de más de uno, se identificaron los segmentos censales segregados y se hicieron las correcciones necesarias.

La base de datos fue desagregada por sexos y por nueve grupos de edad ( $<5$ años, 5-14, 15-24, 25-34, 35-44, 45-54, 55-64, 65-74, $\geq 75$ ), lo que generó un total de casi 130000 observaciones en los 420 distritos durante los 17 años de observación retrospectiva.

Las variables para el análisis fueron de cuatro tipos:

Variables de resultado: número de defunciones por distrito, año, sexo y edad. Se estimaron modelos para la mortalidad de los niños menores de 5 años, adultos de 15 años o más y para la mortalidad según tres grandes grupos de causas: 1) por enfermedades transmisibles o desencadenadas por procesos infecciosos, que se denominarán en adelante simplemente "transmisibles" (diarrea, tuberculosis, infecciones respiratorias, sida, otras enfermedades infecciosas y afecciones parasitarias, así como desnutrición, enfermedades maternas y cáncer cervicouterino); 2) por afecciones de origen social (alcoholismo, accidentes, homicidio y suicidio); y 3) un grupo residual compuesto principalmente por afecciones crónicas y degenerativas. Como fuente de información se utilizaron los archivos electrónicos de datos de defunción del Instituto Nacional de Estadística y Censos (INEC), disponibles en Internet (12).

Variables de exposición: población de cada distrito por año, sexo y grupo de edad, según estimaciones del Centro Centroamericano de Población de la Universidad de Costa Rica (12).

Variables que miden la intervención: una variable que indica si la reforma del sector de la salud se había adoptado en el distrito el año en cuestión (con reforma $=1$, sin reforma $=0$ ); y una variable de intervalo que indica el número de años desde la adopción de la reforma ( $\sin$ reforma $=0$, un año $=1$, dos años $=2$, etc.). Estas variables se crearon a partir del dato del año de inauguración de 84 áreas de salud y la definición de los distritos que integraron cada área. ${ }^{2}$ Por ejemplo, a la variable "reforma" de un distrito perteneciente a un área de salud inaugurada en 1997 se le asignó cero entre los años 1985 y 1996, y uno de 1997 en adelante.

Variables de confusión o de control: se utilizaron cuatro indicadores provenientes de la información anual del registro de nacimientos (12): proporción de nicaragüenses, estimada por la proporción de madres nicaragüenses (esta variable se incluye en el estudio debido al extraordinario auge reciente de la migración nicaragüense a Costa Rica y debido a que muchos sectores atribuyen a esta migración un importante impacto en los servicios y en las tendencias de la salud en el país); tasa de fecundidad general (nacimientos por cada 100 mujeres de 15 a 44 años de edad); proporción de madres adolescentes (menores de 20 años de edad); y proporción de madres solteras. También se emplearon otros cuatro indicadores calculados mediante la interpolación de los datos de los censos de 1984 y 2000: proporción de adultos con estudios secundarios completos o incompletos; proporción de personas con seguro de salud dentro de la población total; índice normalizado de ingresos en el distrito (estimado según la posesión de 11 bienes en los hogares, promediado para el distrito y normalizado a la media y desviación estándar del censo correspondiente, de modo que se expresa como el número de desviaciones estándar con respecto a la media); proporción de personas que se habían trasladado a residir al distrito (desde el exterior o desde otros cantones) en los últimos 5 años.

\section{Modelo de regresión}

El impacto de la reforma se determinó mediante el modelo de regresión múltiple de Poisson (13) con efectos fijos (14), utilizando el paquete de

\footnotetext{
2 Luis Vílchez, Gerencia de Modernización de la CCSS, comunicación personal.
}

cómputo Stata (15). Se estimaron modelos de efectos fijos, ya que estos permiten eliminar los sesgos de selección persistentes en el tiempo mediante la comparación de las variaciones anuales. Se optó por un modelo de regresión de Poisson ya que, como variable dependiente, el número de defunciones tiene una distribución de Poisson que toma valores enteros, positivos y generalmente pequeños. La regresión de Poisson es equivalente a una regresión lineal de los logaritmos de las tasas de mortalidad. Los coeficientes exponenciados permiten estimar el riesgo relativo (RR) de morir. Los errores estándar de los coeficientes se estimaron mediante procedimientos robustos existentes en el paquete Stata, empleando el distrito como variable de conglomeración. Los coeficientes y sus errores estándar se estimaron por máxima verosimilitud. Con los errores estándar se calcularon intervalos de confianza de 95\% (IC95\%) para identificar la significación estadística de los efectos de las variables explicativas en la variable dependiente (mortalidad) en la regresión.

Con los coeficientes de los modelos de regresión se simularon las tasas de mortalidad con y sin reforma para los distritos $\mathrm{y}$, por agregación, para todo el país. Las tasas simuladas "con reforma" son, simplemente, las tasas predichas por el modelo de regresión y los valores observados de las variables explicativas del modelo. Las tasas simuladas "sin reforma" son similares a las anteriores, excepto que la variable correspondiente a la reforma toma el valor cero (sin reforma) en todos los años y distritos.

\section{Acceso a los servicios}

Para explorar los mecanismos del posible impacto de la reforma en la mortalidad, la evaluación se complementó con el análisis del efecto de la reforma en una variable mediadora: el acceso de la población a los servicios de salud del primer nivel de atención. La información para este análisis provino de un estudio previo sobre el acceso físico a estos servicios, realizado a 
CUADRO 1. Características de la población y los servicios según época de la reforma

\begin{tabular}{|c|c|c|c|c|}
\hline \multirow[b]{2}{*}{ Indicadores } & \multicolumn{4}{|c|}{ Población } \\
\hline & Total & $\begin{array}{l}\text { Reforma de } \\
1995-1996\end{array}$ & $\begin{array}{l}\text { Reforma de } \\
\text { 1997-2000 }\end{array}$ & $\begin{array}{c}\text { Sin } \\
\text { reforma }\end{array}$ \\
\hline Población (millones de personas) & 3,81 & 1,27 & 1,31 & 1,23 \\
\hline Personas con educación secundaria & $46 \%$ & $34 \%$ & $49 \%$ & $55 \%$ \\
\hline Inmigrantes nicaragüenses ${ }^{a}$ & $13 \%$ & $12 \%$ & $15 \%$ & $14 \%$ \\
\hline Tasa de mortalidad infantil 1993-1995 & & & & \\
\hline (por 1000 nacidos vivos) & 13,4 & 14,5 & 12,5 & 13,2 \\
\hline Número de EBAIS b en 2001 & 665 & 343 & 264 & 58 \\
\hline
\end{tabular}

a Madres nicaragüenses que aparecen en las estadísticas de nacimientos de 1999 a 2001.

b EBAIS: equipos básicos de atención integral de la salud.

partir de los datos del censo del año 2000 y un inventario de los establecimientos de salud, auxiliado por sistemas de información geográfica (16). Ese estudio propuso varias medidas de acceso. En la presente investigación se utilizó una de las medidas más sencillas y fáciles de interpretar: la equidad en el acceso a los servicios médicos de una comunidad, medida por el porcentaje de personas que no disponen de atención médica (establecimiento con dos o más días semanales de consulta médica) a menos de $4 \mathrm{~km}$ de distancia de su hogar.

\section{RESULTADOS}

Los 3,8 millones de habitantes de Costa Rica empadronados en el censo del año 2000 se pueden dividir en tres grupos aproximadamente iguales, según la época en que sus distritos adoptaron la reforma sanitaria (cuadro 1): el grupo pionero (1995-1996), el grupo intermedio (1997-2000) y el grupo tardío (del 2001 en adelante). La reforma se inició en las zonas periféricas (las más alejadas de la Gran Área Metropolitana de San José), preferentemente en las de menor densidad demográfica, caracterizadas además por tener un menor desarrollo relativo (figura 2). Según los datos censales, $34 \%$ de los adultos de las áreas pioneras tenían educación secundaria completa o incompleta, en comparación con 49\% y 55\% de los otros dos grupos, respectivamente (cuadro 1). Las zonas de mayor concentración poblacional localizadas hacia el centro del país (donde se encuentra la Gran Área Metropolitana) se incorporaron después o aún no lo habían hecho en el momento en que se realizó este estudio.

Los datos muestran, por lo tanto, que la incorporación de las distintas regiones del país a la reforma no ocurrió al azar, sino que hubo una concentración de esfuerzos en las zonas periféricas y en las poblaciones menos favorecidas.

\section{Tendencia de la mortalidad}

Como se indicó en la introducción, indicadores nacionales de esperanza de vida y de mortalidad infantil muestran que a partir de 1995 se reactivaron los avances en la salud del país, después de varios años de estancamiento e, incluso, deterioro. La base de datos del presente estudio permite desagregar las tendencias nacionales para poblaciones diferenciadas según la época de adopción de la reforma. Si las curvas de tendencia mostraran que no hubo progreso en las poblaciones sin reforma y que la reactivación de los avances ocurrió principalmente en las poblaciones con reforma y coincidiendo con la época en que estas la adoptaron, sería un indicio de que la reactivación fue causada por la reforma.

Para observar estas tendencias se agruparon en una gráfica los 420 distritos en cuatro conjuntos, según el año de adopción de la reforma. Además, se agregó la información de los dos sexos y las edades en una sola curva, manteniendo únicamente la distinción entre niños menores de 5 años y adultos (figura 3). Las diferencias en las tendencias no son, sin embargo, claras, aunque se distingue cierta proclividad de las curvas de distritos sin reforma, tanto en niños como en adultos, a una menor disminución de la mortalidad en el período 1995-2000, comparados con los distritos con reforma. El ojo humano obviamente no está en capacidad de discernir si estas diferencias son estadísticamente significativas. Al mismo tiempo, las curvas de tendencia en este análisis gráfico están afectadas por fluctuaciones aleatorias y por variables de confusión que distorsionan la comparación. Para controlar estos factores, medir con precisión las diferencias y establecer su significación estadística (i.e., establecer si las diferencias se deben o no al azar) es necesario pasar del análisis descriptivo de tendencias a análisis estadísticos más poderosos, como la regresión múltiple.

\section{Impacto de la reforma en la mortalidad—análisis de regresión}

Según los resultados del análisis de regresión múltiple de Poisson, la reforma redujo la mortalidad de manera significativa, tanto en niños (8\%) como en adultos (2\%). Los resultados de un segundo análisis de modelación de tipo dosis-respuesta del impacto de los años de reforma mostraron que por cada cinco años adicionales de reforma, la mortalidad de los niños se redujo en $13 \%$ y la de los adultos en $4 \%$ (cuadro 2).

Los efectos de la reforma identificados en la regresión están exentos de los efectos de confusión de las restantes variables en la ecuación, las cuales se muestran también en el cuadro 2 . Ser varón conlleva un riesgo $25 \%$ mayor de morir que ser del sexo femenino en menores de 5 años, y $45 \%$ mayor en personas de 15 años o más. En adultos, cada año de edad se acompañó de un incrementó de $8 \%$ del riesgo de morir. Una mayor tasa de fe- 
FIGURA 3. Tendencias de la mortalidad según el año de adopción de la reforma. Costa Rica, 1985-2001
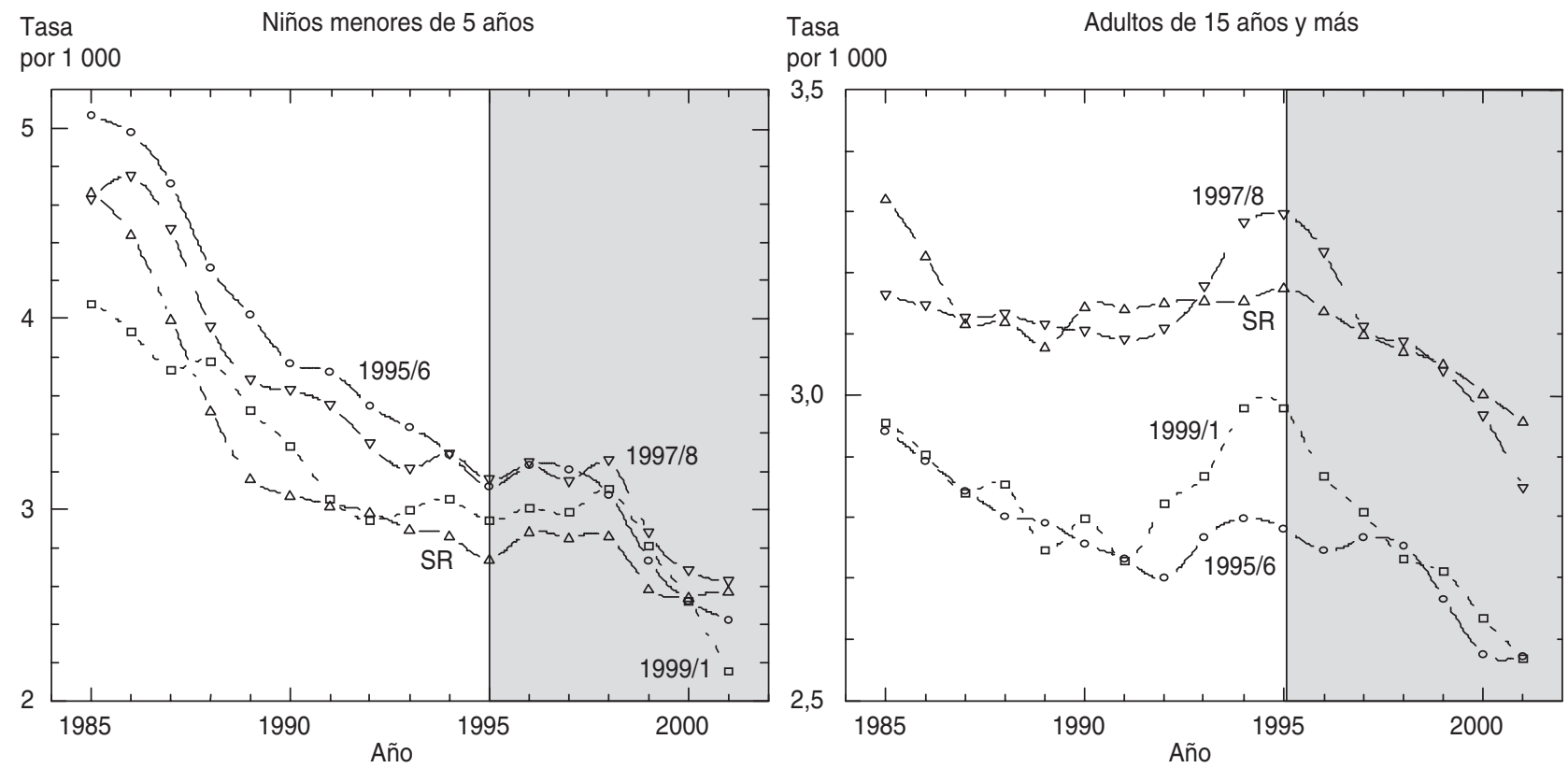

SR $=$ Sin reforma en el año 2002

cundidad en el distrito se asoció de un modo significativo con una mayor tasa de mortalidad, tanto en niños como en adultos, especialmente en los primeros. El nivel educacional en el distrito estuvo asociado con una menor mortalidad únicamente entre los niños, mientras que entre los adultos se observó un aumento de la mortalidad que, aunque pequeño, fue significativo. La inmigración, tanto la nicaragüense como la proveniente de otros cantones del país, no mostró asociación con las variaciones de la mortalidad, como tampoco los indicadores relativos al embarazo en adolescentes y a la fecundidad fuera del matrimonio. El aumento de una desviación estándar en los ingresos promedios de un distrito se vio asociado con una reducción de 5\% de la mortalidad en adultos. La cobertura del seguro social no mostró ninguna asociación con la mortalidad infantil, pero sí una asociación inversa con la mortalidad en adultos.

Al comparar las tasas de mortalidad observadas para toda Costa Rica con las estimadas o predichas mediante la regresión se observó que los modelos lograron captar la tendencia general hacia un aumento de la mortalidad en adultos y hacia la disminución de la mortalidad infantil en el período de 1985 a 2001 (figura 4). La comparación de las líneas obtenidas mediante el modelo de regresión para condiciones

simuladas de ausencia de reforma en todo el país reflejó mayores tasas nacionales de mortalidad a partir de 1995 en esa situación hipotética de que no hubiese ocurrido la reforma. La diferencia entre las dos curvas, expresada en número de defunciones, indica que

CUADRO 2. Modelos de regresión para calcular el impacto de la reforma en la mortalidad. Costa Rica $1985-2001^{\text {a }}$

\begin{tabular}{|c|c|c|}
\hline \multirow[b]{2}{*}{ Variables explicatorias } & \multicolumn{2}{|c|}{ Riesgo relativo de morir (IC95\%) } \\
\hline & Niños (<5 años) & Adultos ( $\geq 15$ años) \\
\hline Reforma & $0,92(0,88-0,97)$ & $0,98(0,96-0,99)$ \\
\hline Sexo masculino & $1,25(1,22-1,29)$ & $1,45(1,44-1,47)$ \\
\hline Edad (por año) & & $1,08(1,08-1,08)$ \\
\hline Personas con seguro ${ }^{\mathrm{b}}(\%)$ & $1,00(0,96-1,04)$ & $1,03(1,01-1,05)$ \\
\hline Personas con educación secundaria ${ }^{b}(\%)$ & $0,77(0,72-0,82)$ & $1,03(1,00-1,06)$ \\
\hline Índice normalizado de ingresos ${ }^{c}$ & $0,97(0,89-1,06)$ & $0,98(0,95-1,01)$ \\
\hline Nicaragüenses ${ }^{b}(\%)$ & $0,99(0,95-1,03)$ & $1,00(0,98-1,01)$ \\
\hline Inmigrantes, últimos 5 años ${ }^{b}(\%)$ & $0,95(0,89-1,02)$ & $0,98(0,95-1,01)$ \\
\hline Tasa general de fecundidad ${ }^{b}(\%)$ & $1,56(1,47-1,66)$ & $1,12(1,10-1,15)$ \\
\hline Madres adolescentes ${ }^{b}(\%)$ & $1,02(0,97-1,07)$ & $1,01(1,00-1,03)$ \\
\hline Madres solteras ${ }^{\mathrm{b}}(\%)$ & $0,99(0,96-1,03)$ & $1,00(0,98-1,01)$ \\
\hline Dosis, 5 años de reformad ${ }^{d}$ & $0,87(0,82-0,92)$ & $0,96(0,94-0,98)$ \\
\hline
\end{tabular}

a Regresión de Poisson de efectos fijos en la mortalidad del distrito.

${ }^{b}$ Efecto del aumento en 10 puntos porcentuales de la variable explicatoria.

" Número de desviaciones estándar en relación con los ingresos medios de los distritos.

"Efecto según los modelos con la variable "años de reforma" en lugar de "reforma (sí/no)". 
FIGURA 4. Simulación de la tendencia de la mortalidad con y sin reforma. Costa Rica, 1985-2001

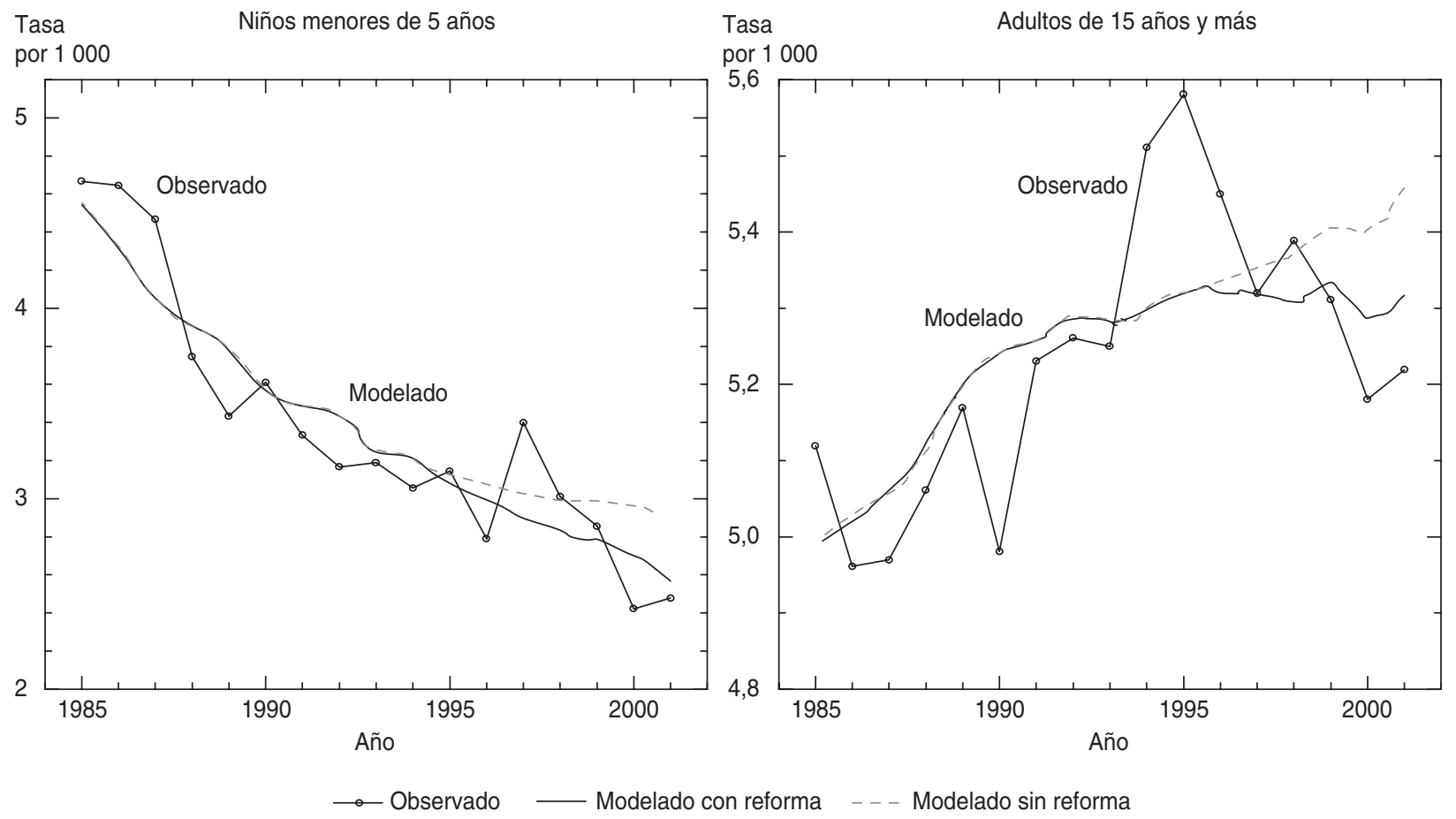

la adopción de la reforma permitió salvar en el año 2001 la vida de 120 niños menores de 5 años y de 350 adultos mayores de 15 años.

\section{Causas de defunción}

¿Mediante qué mecanismos la reforma del sector salud tuvo un impacto en la mortalidad? Una primera aproximación a esta interrogante la proporciona el análisis de las causas de muerte en adultos, según los tres grandes grupos establecidos. ${ }^{3}$ Este análisis mostró que la mortalidad por enfermedades transmisibles, que tradicionalmente son las que más aportaron a la disminución de la mortalidad en el pasado, se redujo en $14 \%$ por efecto de la adopción de la reforma. Un "efecto de dosis" de 5 años de reforma incrementa esta reducción a $22 \%$. En la mortalidad por afecciones crónicas, la más importante en Costa

\footnotetext{
3 Entre los niños no hubo suficientes observaciones para desagregar el análisis por causas de muerte.
}

Rica actualmente, se estimó que la reforma produjo una reducción significativa de $2 \%$, cifra similar al efecto estimado para la mortalidad total en adultos. No se encontró que la reforma hubiese tenido un efecto significativo en la mortalidad por causas de origen social (cuadro 3).

CUADRO 3. Modelos de regresión para calcular el impacto de la reforma en la mortalidad según las causas de muerte. Costa Rica, 1985-2001ª

\begin{tabular}{|c|c|c|c|}
\hline \multirow[b]{2}{*}{ Variables explicativas } & \multicolumn{3}{|c|}{ Riesgo relativo de morir (IC95\%) } \\
\hline & $\begin{array}{l}\text { Enfermedades } \\
\text { transmisibles }\end{array}$ & $\begin{array}{l}\text { Causas } \\
\text { sociales }\end{array}$ & $\begin{array}{l}\text { Afecciones } \\
\text { crónicas }\end{array}$ \\
\hline Reforma & $0,89(0,84-0,95)$ & $1,02(0,98-1,07)$ & $0,98(0,96-1,00)$ \\
\hline Sexo masculino & $0,98(0,94-1,02)$ & $2,08(2,02-2,14)$ & $1,43(1,42-1,45)$ \\
\hline Edad (cada año) & $1,07(1,07-1,07)$ & $1,04(1,04-1,04)$ & $1,09(1,09-1,09)$ \\
\hline Personas con seguro ${ }^{c}(\%)$ & $0,97(0,90-1,04)$ & $1,06(1,01-1,11)$ & $1,03(1,01-1,05)$ \\
\hline Personas con educación secundaria ${ }^{\mathrm{C}}(\%)$ & $1,11(1,00-1,24)$ & $1,04(0,96-1,12)$ & $1,02(0,99-1,06)$ \\
\hline Índice normalizado de ingresos ${ }^{d}$ & $1,00(0,87-1,14)$ & $1,03(0,94-1,13)$ & $0,97(0,94-1,01)$ \\
\hline Nicaragüenses ${ }^{c}(\%)$ & $0,99(0,93-1,04)$ & $0,99(0,96-1,03)$ & $1,00(0,98-1,01)$ \\
\hline Inmigrantes, últimos 5 años ${ }^{c}(\%)$ & $0,95(0,85-1,06)$ & $1,00(0,92-1,08)$ & $0,98(0,95-1,01)$ \\
\hline Tasa general de fecundidad ${ }^{\mathrm{c}}(\%)$ & $1,17(1,07-1,29)$ & $1,16(1,09-1,24)$ & $1,11(1,08-1,14)$ \\
\hline Madres adolescentes ${ }^{c}(\%)$ & $1,02(0,96-1,09)$ & $0,99(0,94-1,03)$ & $1,02(1,00-1,04)$ \\
\hline Madres solteras ${ }^{c}(\%)$ & $1,01(0,96-1,06)$ & $1,00(0,97-1,03)$ & $0,99(0,98-1,01)$ \\
\hline Dosis, 5 años de reforma ${ }^{e}$ & $0,83(0,77-0,90)$ & $1,01(0,95-1,06)$ & $0,96(0,94-0,98)$ \\
\hline
\end{tabular}


CUADRO 4. Población con acceso deficiente a los servicios médicos. Costa Rica, 19942000

\begin{tabular}{lcccc}
\hline & \multicolumn{3}{c}{ Población (\%) } \\
\cline { 2 - 5 } \multicolumn{1}{c}{ Año } & Total & $\begin{array}{c}\text { Reforma } \\
1995-1996\end{array}$ & $\begin{array}{c}\text { Reforma } \\
1997-2000\end{array}$ & $\begin{array}{c}\text { Sin reforma } \\
\text { en junio de 2000 }\end{array}$ \\
\hline 1994 & 22 & 36 & 14 & 14 \\
2000 & 13 & 21 & 6 & 11 \\
Diferencia & -9 & -15 & -7 & -3 \\
\hline
\end{tabular}

\section{Equidad en el acceso a los servicios de salud}

El análisis geográfico de la oferta y la demanda de los servicios de salud mostró una clara asociación entre la reforma y el acceso a los servicios de salud en el primer nivel de atención (cuadro 4). De 1994 a 2000 el porcentaje de la población con acceso deficiente o poco equitativo a los servicios se redujo de 22 a $13 \%$. Esta mejora fue mucho mayor en las áreas pioneras de la reforma, donde el porcentaje de inequidad en el acceso disminuyó en 15\% en comparación con solo $3 \%$ en las áreas que aún no se habían incorporado a la reforma a mediados del año 2000 (cuadro 4).

\section{DISCUSIÓN}

La reforma del sector de la salud es posiblemente el acontecimiento de mayor trascendencia en el campo de la salud pública en Costa Rica en la última década del siglo pasado. La reversión del estancamiento y deterioro en la esperanza de vida observados de 1985 a 1995 parece estar asociada con el proceso de reforma iniciado en 1995.

La circunstancia de que la reforma no se adoptara al mismo tiempo en todo el país creó un cuasiexperimento natural que permitió evaluar el impacto de la reforma con la ayuda de modelos de regresión múltiple. Según estos modelos, la adopción de la reforma resultó en una reducción significativa de la mortalidad de niños menores de 5 años (8\%) y de adultos mayores de 15 años (2\%). El impacto de la reforma resultó en una reducción de la mortalidad de adultos por enfermedades transmisibles o desencadenadas por procesos infecciosos de $14 \%$, de $2 \%$ en la mortalidad por afecciones crónicas, y nulo en la originada por factores sociales. Estos porcentajes son substancialmente mayores después de 5 años de reforma, en una suerte de efecto dosis-respuesta.

La simulación de una situación hipotética de ausencia de reforma indica que la reforma salvó la vida de aproximadamente 120 niños menores de 5 años y de 350 adultos mayores de 15 años solo en el año 2001.

La reforma mostró una mayor asociación con la mortalidad en niños menores de 5 años y con la mortalidad por enfermedades transmisibles en los adultos, lo que apunta a que el efecto de la reforma pudo lograrse mediante la aplicación de medidas y tecnologías sencillas y de bajo costo. La reducción de la mortalidad por enfermedades crónicas en los adultos requiere intervenciones más complejas y costosas, pues hay que actuar sobre factores que han estado latentes durante años o décadas en la vida de las personas. Este resultado es además coherente con el hecho de que la evaluación se concentró en la aplicación de la reforma en el primer nivel de atención.

La falta de una asociación entre la reforma y la mortalidad por causas de origen social es, a su vez, coherente con la situación general de la salud pública en Costa Rica, donde no se ha logrado controlar este problema. De hecho, se observa un aumento de la mortalidad por algunas de estas causas, como el homicidio. Esto puede deberse al enfoque casi exclusivamente médico de la atención de salud y a la falta de respuesta de la medicina ante estos problemas, con la excepción de la atención médica de los heridos en los servicios de emergencia.

El impacto de la reforma documentado en el presente trabajo pudo lograrse posiblemente gracias a su focalización en las regiones menos desarrolladas del país, lo que ayudó a reducir la brecha existente en cuanto a la equidad en el acceso a los servicios de salud.

Los resultados de esta evaluación no son totalmente concluyentes porque no se originan en un diseño experimental aleatorizado. Verdaderos experimentos son, empero, una utopía en intervenciones de salud pública de la magnitud de la reforma analizada (aunque no son imposibles de efectuar). Los resultados obtenidos pueden considerarse, sin embargo, muy robustos y de alta validez, porque provienen de lo que podría llamarse el mejor diseño evaluativo después del experimento aleatorizado: un diseño cuasiexperimental, con mediciones antes y después de la reforma, con grupos de intervención y testigos, y con control de los factores de confusión mediante el análisis de múltiples variables. La presencia de efectos del tipo dosis-respuesta es un elemento que refuerza la validez de los resultados obtenidos.

La falta de aleatoriedad en la selección de las áreas intervenidas podría dar lugar a sesgos que distorsionen la evaluación en cualquier dirección. Por una parte, podría producirse un sesgo "de innovación", es decir, que las primeras comunidades que adoptaron la reforma podrían ser más propensas a innovar en otras áreas. Esto podría llevar a que esas comunidades tengan mejores resultados en materia de salud y a que los logros generados por esta propensión a innovar se atribuyan erróneamente a la reforma. Por otra parte, la focalización de la intervención en áreas con mayores necesidades o carencias de salud podría generar un sesgo de selección en la dirección opuesta, es decir, que la intervención podría aparecer asociada con resultados menos favorables en materia de salud. Estos posibles sesgos de selección se neutrali- 
zaron en gran parte en el presente estudio mediante el control estadístico de los efectos de las variables de confusión en la regresión múltiple y el uso de mediciones antes y después de la intervención a fin de comparar tendencias en vez de niveles.

Otro sesgo potencial podría deberse a un "efecto de derrame" o "de cascada". Las áreas sin intervención podrían haberse contaminado de ciertos aspectos de la reforma. Por ejemplo, en muchas zonas comenzaron a establecerse los EBAIS antes de la fecha oficial de adopción de la reforma. Otro ejemplo es que las mejoras gerenciales y administrativas adoptadas en el nivel central como parte de la reforma podrían haber beneficiado a las regiones que oficialmente no la habían adoptado. El sesgo de estos efectos de derrame consistiría, sin embargo, en reducir parte del impacto observable. Las áreas sin reforma tomadas como referencia tendrían una mejor salud gracias a este efecto indirecto de la reforma, de tal manera que el impacto pudo haber sido mayor que el cuantificado en este estudio.

Los datos analizados permiten concluir que la focalización inicial de la reforma en zonas menos desarrolladas permitió mejorar el grado de equidad en el acceso a los servicios médicos y redujo significativamente la mortalidad. La reforma puso fin a una década de estancamiento en el mejoramiento de algunos indicadores en Costa Rica, como la esperanza de vida y la mortalidad.

La conclusión anterior requiere, empero, varias aclaraciones. En primer lugar, estos resultados reflejan exclusivamente las condiciones de Costa Rica; no se deben extrapolar a otros contextos debido a la variación existente en los diferentes países acerca de lo que se entiende por reforma del sector de la salud. Por ejemplo, en algunos casos ha sido simplemente una etiqueta de la privatización de los servicios, mientras que en otros, la reforma ha sido sinónimo de descentralización, con las particularidades propias de cada país.

En segundo lugar, el presente estudio valoró solamente el impacto de un as- pecto de la reforma costarricense: los cambios en el primer nivel de atención provocados por el establecimiento de las áreas de salud y la apertura de EBAIS. No evaluó el impacto de los cambios introducidos por la reforma en los niveles superiores de atención y en el sistema de salud en su conjunto. El hecho de que los cambios en el primer nivel de atención hayan tenido un impacto favorable no significa que los ocurridos en otros niveles lo tengan también.

Debe notarse, finalmente, que la presente evaluación fue posible debido a la existencia en Costa Rica de un adecuado sistema de información independiente de la reforma. Lamentablemente, el programa de reforma del sector de la salud no previó establecer un sistema de evaluación y seguimiento ni de generación de información que permitiera valorar su impacto. La falta de esta información, así como la carencia de indicadores de base, de proceso y de resultados en conexión con las distintas actividades, limitan las posibilidades de realizar una evaluación más profunda.

\section{REFERENCIAS}

1. Population Reference Bureau. World population data sheet 2001. Washington, D.C.: Population Reference Bureau; 2001.

2. Arias E. United States life tables, 2000. National Vital Statistics Reports. 2002;51(3):1-40.

3. Rosero-Bixby L. Socioeconomic development, health interventions, and mortality decline in Costa Rica. Scand J Soc Med. 1991;Suppl 46: 33-42.

4. Mesa-Lago C. Health care in Costa Rica: boom and crisis. Soc Sci Med. 1985;21(1):13-21.

5. Rosero-Bixby L. Tendencias en la mortalidad y esperanza de vida. Costa Rica 1970-2001. San José, Costa Rica: Proyecto Estado de la Nación. (En prensa).

6. Costa Rica, MIDEPLAN, Ministerio de Salud. Plan Nacional de Reforma del Sector Salud. San José: Presidencia de la República, Programa de Reforma del Estado; 1993.

7. Pan American Health Organization. Profile of the health services system of Costa Rica. 2nd ed. Washington, D.C.: PAHO, Division of Health Systems and Services Development;
2002. Hallado en: http://www.americas. health-sector-reform.org/english/cor-preng2ed.pdf. Acceso el 8 de diciembre de 2003.

8. World Bank. Costa Rica: second health sector strengthening and modernization project. Washington, D.C.: World Bank; 2001. (World Bank Report No. PID10534).

9. Sáenz L. Reforma del sector salud en Costa Rica: antecedentes, objetivos y algunos hallazgos. San José: Proyecto Estado de la Nación. (En prensa).

10. Proyecto Estado de la Nación. Estado de la nación en desarrollo humano sostenible: octavo informe 2001. San José, Costa Rica: Proyecto Estado de la Nación; 2002.

11. Mohr LB. Impact analysis for program evaluation. Chicago: Dorsey Press; 1988.

12. Centro Centroamericano de Población, Universidad de Costa Rica. Estadísticas vitales del 2002. En: Consulta a censos y grandes bases estadísticas [sitio en Internet]. Hallado en: http://censos.ccp.ucr.ac.cr. Acceso el 8 de diciembre de 2003.
13. Cameron AC, Trivedi PK. Regression analysis of count data. Cambridge: Cambridge University Press; 1998.

14. Rosenzweig MR, Wolpin KI. Evaluating the effects of optimally distributed public programs. Am Econ Rev. 1986;76:470-482.

15. Stata Corporation. Stata statistical software: release 7.0. College Station, Texas: Stata Corporation; 2001.

16. Rosero-Bixby L. Spatial access to health care in Costa Rica and its equity: a GIS-based study. Soc Sci Med. (En prensa).

Manuscrito recibido el 4 de abril de 2003. Aceptado para publicación, tras revisión, el 3 de diciembre de 2003. 
ABSTRACT Objective. To assess the impact of health sector reform in Costa Rica on that country's child and adult mortality rates and on the people's access to primary health care. Methods. Health sector reform was initiated in Costa Rica in 1995 in some districts,

\section{Assessing the impact of health sector reform in Costa Rica through a quasi-experimental study}

but in others reforms were adopted later. This made it possible to perform a time series analysis, using a quasi-experimental study design, in which observations were made annually from 1985 through 2001 for each of the 420 districts that existed in Costa Rica in 1984. The time series were divided into three periods that allowed all districts to be grouped into three categories (pioneer, intermediate, and late) according to the year when they first implemented health sector reform: 1995-1996; 19972000; and 2001 or after, respectively. For each of these periods, mortality rates were broken down by cause (communicable, socially-determined, or chronic disease), sex, and age group. The status of the reform process in a particular district was described by two indicators: (1) the presence or absence of health sector reform during a given period and, wherever such reforms had been adopted, (2) the number of years that had transpired since their adoption. Eight variables were used to control for confounders. Vital statistics and demographic data were obtained from the National Institute for Statistics and Census' [Centro Nacional de Estadística y Censos] electronic database.

Poisson multiple regression analysis with fixed effects was used to estimate the impact of reform on child and adult mortality from different causes. Assessment of the population's access to primary care before and after the reform was based on the percentage of people who lived within a $4 \mathrm{~km}$ radius of a health facility that offered patient visiting hours two or more days a week. This information came from a previous study that used census data from 2000 and geographic information systems to map health care facilities throughout the country.

Results. Multiple regression showed that the reform was associated with an overall $8 \%$ reduction in deaths among children and with a $2 \%$ reduction in deaths among adults, both statistically significant. Also noted were a $14 \%$ reduction in deaths from communicable diseases or from conditions brought on by the presence of infectious processes, a $0 \%$ reduction in deaths from socially-determined causes, and a $2 \%$ reduction in deaths from chronic diseases. An estimated 120 child lives and 350 adult lives were saved by the reform in 2001 alone. Also, the percentage of people without equitable access to primary health services dropped by 15\% between 1994 and 2000 in areas where health sector reform was implemented in 1995-1996, whereas areas that had not yet initiated health sector reform in 2000 experienced only a 3\% reduction.

Conclusion. Health sector reform significantly reduced mortality in Costa Rica and put an end to a decade of stagnation, as shown by certain health indicators, such as life expectancy. Equity in access to primary care improved considerably, perhaps because the first reforms were implemented in less developed areas of the country. 